\title{
Counterrotating Nuclear Disks in Arp 220
}

\section{Citation}

Sakamoto, K., N. Z. Scoville, M. S. Yun, M. Crosas, R. Genzel, and L. J. Tacconi. 1999.

"Counterrotating Nuclear Disks in Arp 220." The Astrophysical Journal 514 (1): 68-76. https:// doi.org/10.1086/306951.

\section{Permanent link}

http://nrs.harvard.edu/urn-3:HUL.InstRepos:41399838

\section{Terms of Use}

This article was downloaded from Harvard University's DASH repository, and is made available under the terms and conditions applicable to Other Posted Material, as set forth at http:// nrs.harvard.edu/urn-3:HUL.InstRepos:dash.current.terms-of-use\#LAA

\section{Share Your Story}

The Harvard community has made this article openly available.

Please share how this access benefits you. Submit a story.

\section{Accessibility}


THE ASTROPHYSICAL JOURNAL, 514:68-76, 1999 March 20

(C) 1999. The American Astronomical Society. All rights reserved. Printed in U.S.A.

\title{
COUNTERROTATING NUCLEAR DISKS IN ARP 220
}

\author{
K. Sakamoto, ${ }^{1}$ N. Z. Scoville, ${ }^{1}$ M. S. Yun, ${ }^{2}$ M. Crosas, ${ }^{3}$ R. Genzel, ${ }^{4}$ and L. J. Tacconi ${ }^{4}$ \\ Received 1998 May 26; accepted 1998 October 28
}

\begin{abstract}
The ultraluminous infrared galaxy Arp 220 has been observed at 0.5 resolution in CO (2-1) and $1 \mathrm{~mm}$ continuum using the newly expanded Owens Valley Millimeter Array. The CO and continuum peaks at the double nuclei and the surrounding molecular gas disk are clearly resolved. We find steep velocity gradients across each nucleus $\left(\Delta V \sim 500 \mathrm{~km} \mathrm{~s}^{-1}\right.$ within $r=0$ ".3) whose directions are not aligned with each other and with that of the outer gas disk. We conclude that the double nuclei have their own gas disks $(r \sim 100 \mathrm{pc})$, are counterrotating with respect to each other, and are embedded in the outer gas disk $(r \sim 1 \mathrm{kpc})$ rotating around the dynamical center of the system. The masses of each nucleus are $M_{\text {dyn }} \gtrsim 2 \times 10^{9} M_{\odot}$, based on the CO kinematics. Although there is no evidence of an old stellar population in the optical or near-infrared spectroscopy of the nuclei (probably owing to the much brighter young population), it seems likely that these nuclei were "seeded" from the premerger nuclei in view of their counterrotating gas kinematics. The gas disks probably constitute a significant fraction $(\sim 50 \%)$ of the mass in each nucleus. The $\mathrm{CO}$ and continuum brightness temperatures imply that the nuclear gas disks have high area filling factors $(\sim 0.5-1)$ and extremely high visual extinctions $\left(A_{V} \simeq 1000\right.$ mag). The molecular gas must be hot $(\geq 40 \mathrm{~K})$ and dense $\left(\geq 10^{4-5} \mathrm{~cm}^{-3}\right)$, given the large mass and small scale height of the nuclear disks. The continuum data suggest that the large luminosity (be it starburst or active galactic nucleus) must originate within $\lesssim 100 \mathrm{pc}$ of the two nuclear gas disks, which were presumably formed through concentration of gas from the progenitor outer galaxy disks.
\end{abstract}

Subject headings: galaxies: evolution - galaxies: individual (Arp 220) - galaxies: interactions galaxies: ISM

\section{INTRODUCTION}

Arp 220 is the prototype ultraluminous infrared galaxy with $L_{8-1000 \mu \mathrm{m}}=1.4 \times 10^{12} L_{\odot}$ (Soifer et al. 1987). The galaxy is thought to be in the final stage of merging since optical images show extended tidal tails (Arp 1966), and radio and near-infrared (NIR) imaging reveal double nuclei with a projected separation of $\sim 300$ pc (Norris 1985; Graham et al. 1990). Arp 220 also has a massive concentration of molecular gas $\left(\sim 10^{10} M_{\odot}\right)$ within the central kiloparsec (Scoville et al. 1986). These characteristics are similar to those of many of the ultraluminous infrared galaxies, which often show morphological evidence of strong interactions or merging (Sanders et al. 1988) and have large molecular gas masses $\left(\sim 10^{10} M_{\odot}\right.$; Sanders, Scoville, \& Soifer 1991; Solomon et al. 1997). The concentration of molecular gas in the nucleus is in accord with gasdynamical simulations of galaxy mergers (see, e.g., Barnes \& Hernquist 1991).

High-resolution observations of molecular gas and dust continuum can provide crucial information on gasdynamics and thermal structure in the nuclear region of Arp 220. Arcsecond resolution observations by Scoville, Yun, \& Bryant (1997; hereafter SYB97) revealed a kiloparsec-size molecular gas disk rotating around the double nucleus. Peaks in the CO line and millimeter-wave continuum at the two nuclei in the kpc-scale disk were suggested by SYB97 and confirmed by Downes \& Solomon (1998; hereafter

\footnotetext{
${ }^{1}$ California Institute of Technology, 105-24, Pasadena, CA 91125; ks@astro.caltech.edu.

${ }_{2}^{2}$ National Radio Astronomy Observatory, P.O. Box 0, Soccoro, NM 87801-0387.

${ }^{3}$ Harvard-Smithsonian Center for Astrophysics, 60 Garden Street, Cambridge, MA 02138.

${ }^{4}$ Max-Planck-Institut für Extraterrestrische Physik, D-85740, Garching, Germany.
}

DS98). High-resolution near-infrared imaging with the near-infrared camera and multiobject spectrometer (NICMOS) onboard the Hubble Space Telescope (HST) shows an area of extremely high dust obscuration south of the western nucleus, which is interpreted as a thin, opaque dust disk embedded in the central star cluster (Scoville et al. 1998). The formation of these nuclear disks during a galactic merger may occur naturally as a result of the high dissipation rates in the dense gas, but their role in the promotion of nuclear starbursts and feeding a preexisting active galactic nucleus (AGN; specifically the source of the large farinfrared, or FIR, luminosity) remains very ill defined. However, given the fact that the molecular gas in the nucleus is by far the largest reservoir of an interstellar medium (ISM) available for both star formation and nuclear accretion, it is likely that the nuclear disks play a central role in the nuclear activity. In this paper, we report 0.5 resolution observations of molecular gas and dust in Arp 220 with the goal of better defining both the disk structure and the kinematics of the nuclei. We adopt the distance of $77 \mathrm{Mpc}$ for Arp 220, at which 1" corresponds to $373 \mathrm{pc}$.

\section{OBSERVATIONS}

Aperture synthesis observations were made on 1998 March 19 using the Owens Valley Millimeter Array. The six $10 \mathrm{~m}$ antennas were deployed in the new $\mathrm{U}$ (ultra-high) configuration that has $400 \mathrm{~m}$ (east-west) and $330 \mathrm{~m}$ (northsouth) baselines. Two SIS receivers were used simultaneously to observe the $\mathrm{CO}(J=2-1)$ line and the 1.3 and 3.0 $\mathrm{mm}$ continuum. Gains and passbands were calibrated with observations of the quasars $1611+343$ and $3 \mathrm{C} 273$, respectively. The flux scale for the quasars was established by 


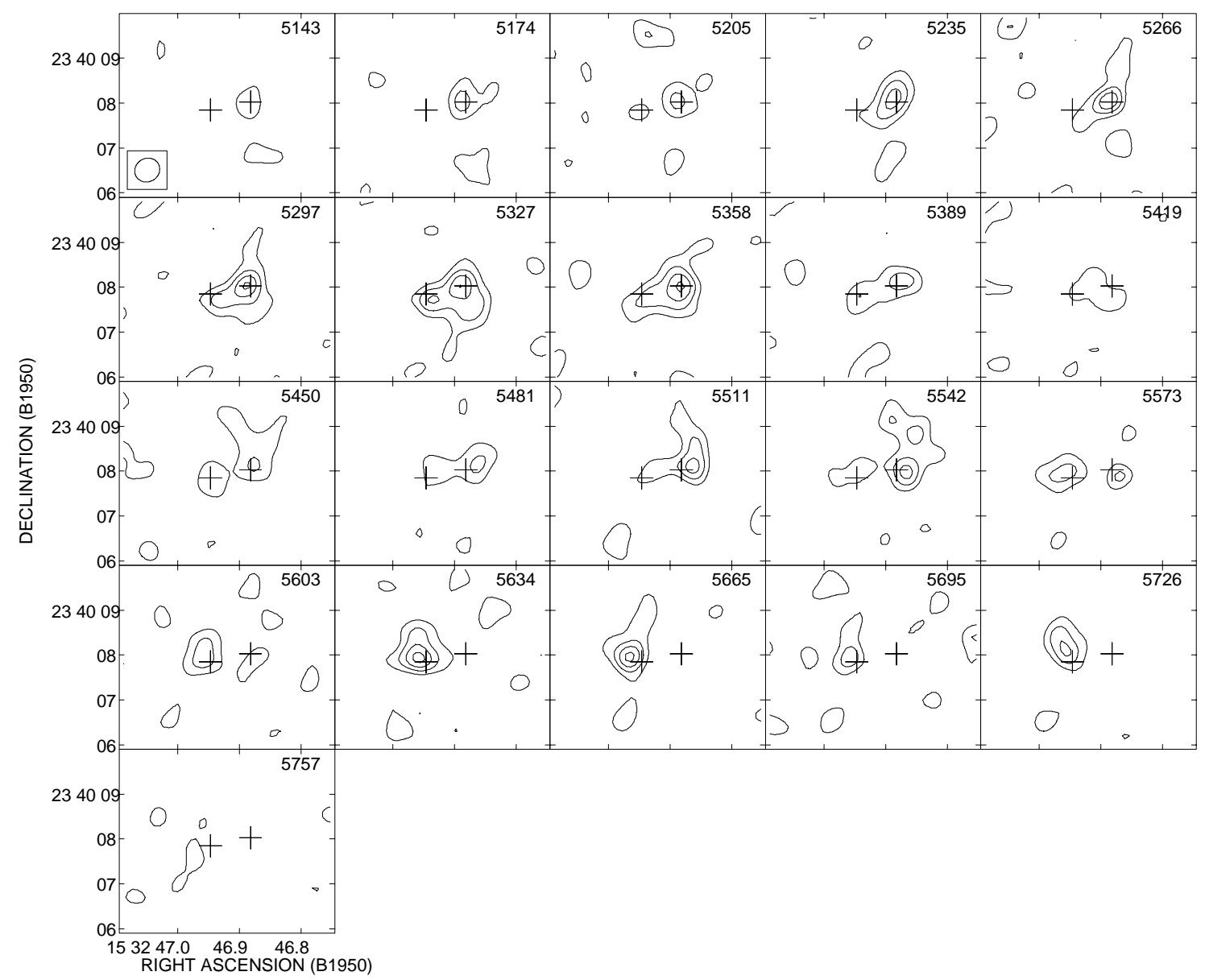

FIG. 1. - CO (2-1) continuum-subtracted channel maps from the U-array data. Contours are at $2,3,4, \ldots, 8 \times 46 \mathrm{mJy}^{\text {beam }}{ }^{-1}(1 \sigma)$. Crosses show the positions of the continuum peaks. Velocities in $\mathrm{km} \mathrm{s}^{-1}$ are shown in the northwest corner of all panels. The synthesized beam $\left(0{ }^{\prime \prime} .57 \times 0\right.$ " $52 \mathrm{FWHM}$, P.A. $=-60^{\circ}$ ) is shown in the first panel.

observing Neptune ${ }^{5}$ and is accurate to $\sim 10 \%$. The derived flux densities of $1611+343$ were $1.4 \mathrm{Jy}$ at $1.3 \mathrm{~mm}$ and $2.3 \mathrm{Jy}$ at $3 \mathrm{~mm}$. The digital spectrometers were configured identically to SYB97 in order to span the very broad CO line $\left(\Delta V=900 \mathrm{~km} \mathrm{~s}^{-1}\right)$ and to facilitate combination of this data with the earlier $1^{\prime \prime}$ resolution data. Continuum data were recorded from upper and lower sidebands for both 1 and $3 \mathrm{~mm}$ in a $1 \mathrm{GHz}$ bandwidth analog continuum correlator.

The raw data were calibrated with the OVRO/MMA software (Scoville et al. 1993), and maps were processed using the NRAO $^{6}$ AIPS package. The continuum in the image sideband was subtracted from the line data in the $u-v$ domain. Twenty-one channel maps, each of $30.7 \mathrm{~km} \mathrm{~s}^{-1}$ width, were CLEANed and then combined for the moment maps. We have made two sets of maps; one from only the U-array data and the other from the U-array data plus the L- and H-array data taken by SYB97 (which we call LHUarray maps). The former maps filter out most of the extended emission, while the latter retains it; the minimum physical baseline lengths are 100 and $15 \mathrm{~m}$, respectively.

\footnotetext{
${ }^{5}$ Flux observations were made in a separate track to avoid the known CO absorption in the Neptune atmosphere.

${ }^{6}$ The National Radio Astronomy Observatory (NRAO) is a facility of the National Science Foundation operated under cooperative agreement by Associated Universities, Inc.
}

\section{RESULTS}

CO channel maps from the U-array data are shown in Figures 1 and 2, respectively. Figure 3 shows the integrated intensity and mean velocity maps from U- and LHU-array data. The main features in the maps are largely consistent with those in SYB97 and DS98, including the CO and continuum peaks at the east and west nuclei, which we call $\mathrm{E}$ and $\mathrm{W}$, and the $\mathrm{CO}$ disk about $5^{\prime \prime}$ in extent with a velocity gradient across it, approximately along the major axis.

Measured parameters of the $\mathrm{CO}$ and continuum emission are summarized in Table 1. The separation and position angle of the two nuclei, $0^{\prime \prime} .90$ and $101^{\circ}$, are in excellent agreement with those at other wavelengths (Norris 1985; Graham et al. 1990; Scoville et al. 1998). Based on the single-dish line and continuum fluxes reported by Radford, Solomon, \& Downes (1991) and Carico et al. (1992), respectively, we conclude that the LHU maps recover the entire $\mathrm{CO}$ and continuum flux from Arp 220. The U-array maps also fully recover the entire continuum flux (within the $\sim 15 \%$ calibration uncertainties), but only $30 \%$ of total CO line flux.

\section{CONTINUUM EMISSION}

The $1 \mathrm{~mm}$ continuum is predominantly thermal emission from dust (see, e.g., Scoville et al. 1991), i.e., the long wavelength tail of the FIR emission component containing the bulk of the FIR luminosity of Arp 220. The continuum map 


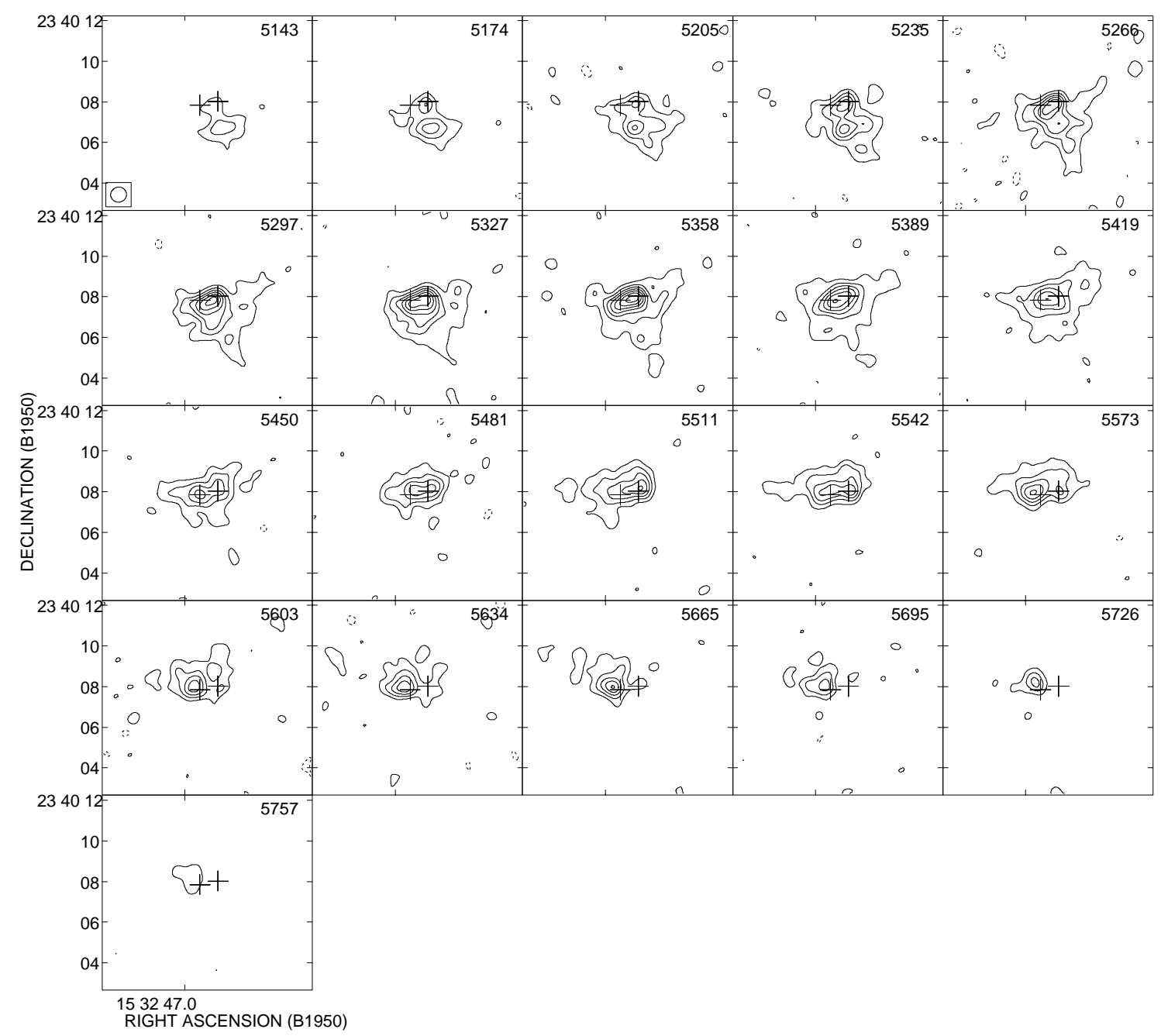

FIG. 2.- CO (2-1) continuum-subtracted channel maps from the LHU-array data. Contours are in steps of $90 \mathrm{mJy}^{\mathrm{beam}}{ }^{-1}(3 \sigma)$. Crosses show the positions of the continuum peaks. Velocities in $\mathrm{km} \mathrm{s}^{-1}$ are shown in the northwest corner of all panels. The synthesized beam $\left(00^{\prime \prime} 78 \times 0\right.$ ".74 FWHM, P.A. $\left.=-80^{\circ}\right)$ is shown in the first panel.

in Figure 3 clearly shows that most of the dust emission arises from the two nuclear components, with less than $20 \%$ contained in a more extended source (as noted above, this map recovers the entire $1.3 \mathrm{~mm}$ continuum flux). The continuum flux ratio between the $\mathrm{E}$ and $\mathrm{W}$ nuclei is about $1: 2$ at both 1 and $3 \mathrm{~mm}$. The spectral slope of each nucleus between 1.3 and $3.0 \mathrm{~mm}$ is $S_{v} \propto v^{3.3 \pm 0.3}$ after subtraction of synchrotron emission, as is discussed in Scoville et al. (1991). This long-wavelength spectrum is consistent with the emission from optically thin dust with an emissivity $\epsilon \propto v^{1.3}$ at $T \simeq 42 \mathrm{~K}$ (see, e.g., Scoville et al. 1991, who modeled the total east plus west flux).

We find no evidence for the extended continuum emission reported by DS98. Their $1.3 \mathrm{~mm}$ fluxes attributed to the $\mathrm{E}$ and $\mathrm{W}$ nuclei are only $45 \%$ and $60 \%$ of our values, but their total flux is $84 \%$ of that reported by us. The smaller fluxes from the nuclei in DS98 are very likely due to their subtraction of an extended "background" or disk component, but the method of separation is not described by them. Their published map shows no obvious extended component, and the total flux measured by us in the $\mathrm{E}$ and W nuclei is $208 \mathrm{mJy}$. Carico et al. (1992) report a single dish flux of $226 \pm 10 \mathrm{mJy}$ at $1.25 \mathrm{~mm}$, which translates to 213 $\mathrm{mJy}$ assuming an emissivity spectral index of 1.3.
The continuum sources are extremely compact. The W nucleus has a deconvolved size of $0.3 \times 0.2(120 \times 70 \mathrm{pc})$, and the $\mathrm{E}$ nucleus is unresolved by our 0 ". 5 beam (i.e., $\leq$ 0 ".2). The equivalent blackbody temperatures for a disk of 0 ".3 diameter are 29 and $51 \mathrm{~K}$ for $\mathrm{E}$ and $\mathrm{W}$ nuclei, respectively. Since the emission probably does not fill the maximum source area, the true brightness temperatures must be higher than these values. A single component fit to the overall FIR spectral energy distribution (SED) of Arp 220 yields a dust temperature of $42 \mathrm{~K}$; however, this low temperature is difficult to reconcile with the minimum brightness temperatures estimated above and the requirement that the dust be optically thin at $1 \mathrm{~mm}$. Thus the overall spectral energy distribution should be modeled more realistically with a range of dust temperatures (mostly higher than $42 \mathrm{~K}$ ), and probably with an emissivity law steeper than $v^{1.3}$. The total dust mass of Arp 220 estimated assuming a single, optically thin component is $\sim 5 \times 10^{7}$ $M_{\odot}($ Scoville et al. 1991). This is probably an underestimate if multicomponent models with moderate opacities are more realistic.

\section{MOLECULAR GAS DISTRIBUTION AND KINEMATICS}

The CO emission peaks at the two continuum nuclei, but 

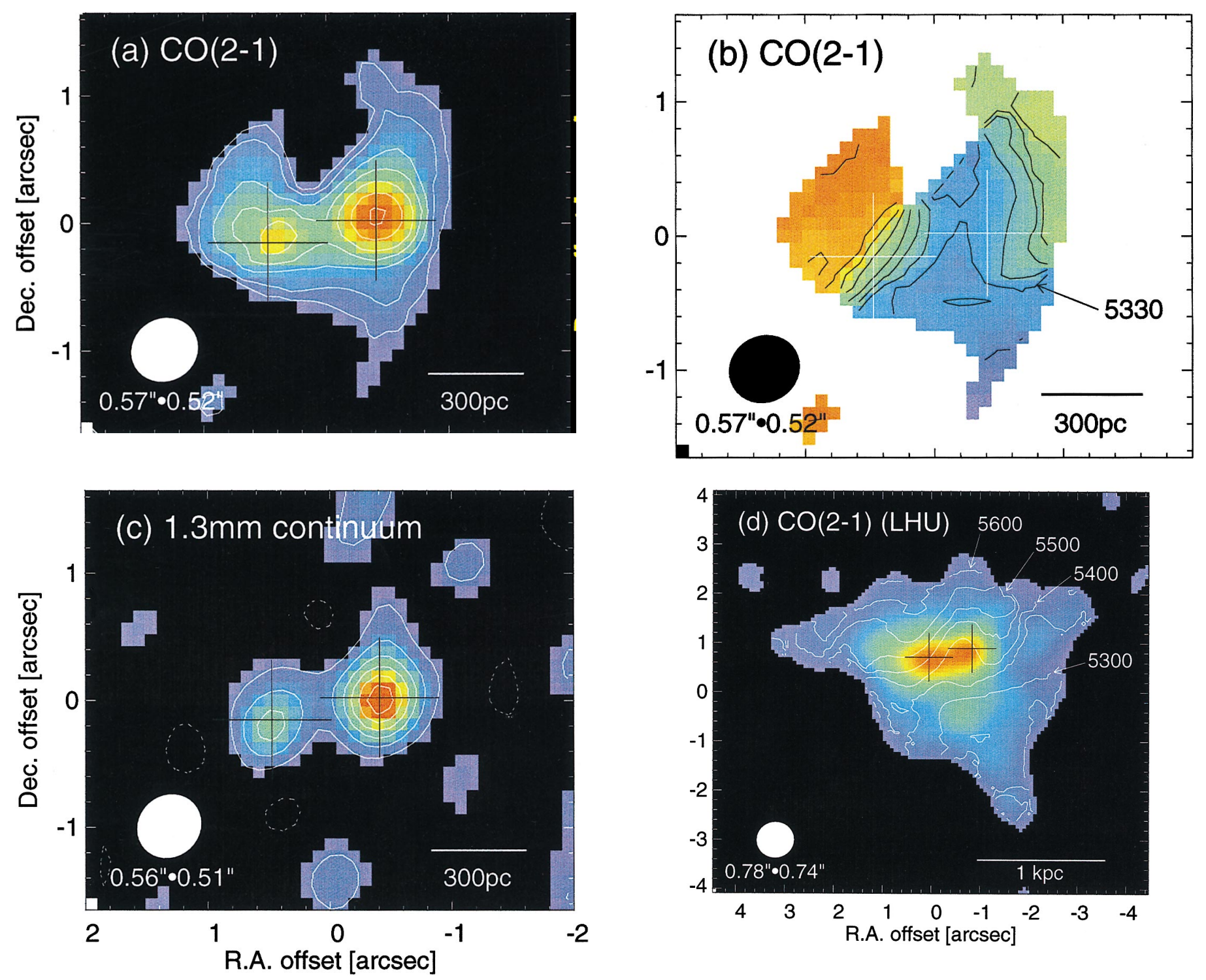

Fig. 3. - Central region of Arp 220. Crosses in each panel indicate the $1.3 \mathrm{~mm}$ continuum positions of the nuclei. (a) Continuum-subtracted CO (2-1) emission integrated over $645 \mathrm{~km} \mathrm{~s}^{-1}$. Contours are at $8.5 \times(1,2,4,6,8,10,12) \mathrm{Jy} \mathrm{beam}{ }^{-1} \mathrm{~km} \mathrm{~s}^{-1}$. (b) Mean velocity of CO (2-1) with contour intervals of 50 $\mathrm{km} \mathrm{s}^{-1}$. (c) $1.3 \mathrm{~mm}$ continuum with contour steps of $18.5 \mathrm{mJy}$ beam ${ }^{-1}(2 \sigma)$ and negative contours (dashed lines). (d) CO (2-1) integrated intensity map and isovelocity contours $\left(50 \mathrm{~km} \mathrm{~s}^{-1}\right.$ increments) made from L, H, and U configuration data. The peak integrated intensity is $193 \mathrm{Jy} \mathrm{beam}^{-1} \mathrm{~km} \mathrm{~s}^{-1}$.

is much more extended than the continuum emission. The total extent of $\mathrm{CO}$ in Figure $3 d$, in which most of the singledish flux is recovered, is about $2 \mathrm{kpc}$. The overall northeastsouthwest velocity gradient seen in this larger scale feature suggests that the gas is rotating as a disk, as modeled by SYB97. The position angle of the velocity gradient, i.e., the major axis of the disk, is measured to be $\sim 25^{\circ}$. It is perpendicular to the symmetric axis (P.A. $\sim 105^{\circ}$ ) of the bipolar $\mathrm{H} \alpha$ emission of Arp 220, which is in the shape of a "doublebubble" of total extent $\sim 24 \times 10 \mathrm{kpc}$, with one bubble on each side of the nucleus (Heckman, Armus, \& Miley 1987). The bubbles have an expansion velocity of a few $100 \mathrm{~km} \mathrm{~s}^{-1}$ and blueshifted (redshifted) on the northwest (southeast) of the nucleus (Heckman, Armus, \& Miley 1990). If the kpcscale molecular disk collimated the outflow of ionized gas, which was attributed to a starburst-driven superwind (Heckman et al. 1987, 1990), then the orientation of the disk is such that the near side of the disk is to the southeast.

About $30 \%$ of the total CO emission is intimately associated with the two nuclei, as revealed most clearly in the
$\mathrm{U}$-array images. The continuum and $\mathrm{CO}$ peaks in the U-array map coincide within 0.05 . The systemic velocities of the two CO peaks (Table 1) agree well with those measured at the two NIR nuclei with the $\mathrm{Br} \gamma$ line (Larkin et al. 1995). The presence of two compact disk components associated with each of the double nuclei was suggested by SYB97 (see their Fig. 11). DS98 clearly demonstrated these peaks but did not interpret them as disks (see below). One of the two models proposed by SYB97 assumed that the gas distribution was smooth and axisymmetric. These assumptions are not valid in the central part of the nuclear disk, i.e., within a diameter equal to the separation of the double nuclei. Instead, their alternative model with peaks on each of the double nuclei is more appropriate based on our higher resolution maps. It was also noted by SYB97 that the $\mathrm{CO}$ emission profile on each nucleus was "double-horned" with a line width of $\sim 250 \mathrm{~km} \mathrm{~s}^{-1}$, suggestive of disks rotating within each nucleus. The two rotating gas disks were also inferred from single-dish profiles of $\mathrm{HCN}$ and $\mathrm{HCO}^{+}$ lines (Taniguchi \& Shioya 1998). The rotating minidisk 
TABLE 1

PARAMETERS OF ARP 220

\begin{tabular}{|c|c|c|c|}
\hline Parameter & Arp $220 \mathrm{E}$ & Arp $220 \mathrm{~W}$ & Total \\
\hline Position $(\Delta \alpha, \Delta \delta)^{\mathrm{a}}(\operatorname{arcsec}) \ldots \ldots \ldots \ldots \ldots \ldots$ & $(0.89,-0.18)$ & $(0,0)$ & \\
\hline 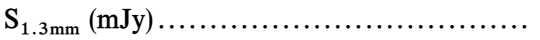 & 66 & 142 & $208^{b}$ \\
\hline Deconvolved size at $1.3 \mathrm{~mm}(\mathrm{pc}) \ldots \ldots \ldots \ldots$ & Unresolved & $120 \times 70\left(\right.$ P.A. $\left.170^{\circ}\right)$ & $\ldots$ \\
\hline$\Delta T_{\text {equiv, } 1.3 \mathrm{~mm}}(\mathrm{~K})^{\mathrm{c}} \ldots \ldots \ldots \ldots \ldots \ldots \ldots \ldots \ldots \ldots \ldots \ldots \ldots \ldots \ldots$ & 29 & 51 & $\ldots$ \\
\hline 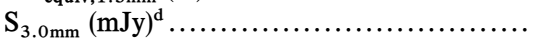 & $(9.5)$ & (19) & 34 \\
\hline $\mathrm{S}_{\mathrm{CO}(2-1)}$ (OVRO U-array) $\left(\mathrm{Jy} \mathrm{km} \mathrm{s}^{-1}\right) \ldots \ldots$ & 120 & 187 & $307^{\mathrm{e}}$ \\
\hline$\Delta T_{b, \mathbf{C O}(2-1)}^{\text {peak }}(\mathrm{K})^{\mathrm{f}} \ldots \ldots \ldots \ldots \ldots \ldots \ldots \ldots \ldots \ldots \ldots \ldots \ldots \ldots \ldots$ & 38 & 37 & $\ldots$ \\
\hline 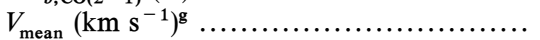 & 5523 & 5350 & $\ldots$ \\
\hline P.A. of velocity gradient $(\mathrm{deg})^{\mathrm{h}} \ldots \ldots \ldots \ldots \ldots$ & 52 & 263 & 25 \\
\hline$\Delta V(r \leq 0,3)\left(\mathrm{km} \mathrm{s}^{-1}\right)^{\mathrm{i}} \ldots \ldots \ldots \ldots$ & 540 & $>480$ & $\ldots$ \\
\hline 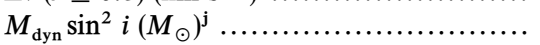 & $1.9 \times 10^{9}$ & $>1.5 \times 10^{9}$ & $5.4 \times 10^{9}$ \\
\hline 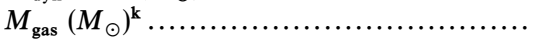 & $\sim 10^{9}$ & $\sim 10^{9}$ & $\ldots$ \\
\hline
\end{tabular}

${ }^{\text {a }}$ Arp $220 \mathrm{~W}$ is at R.A. $=15^{\mathrm{h}} 32^{\mathrm{m}} 46^{\mathrm{s}} .88$, Decl. $=+23^{\circ} 40^{\prime} 08^{\prime \prime} 0(\mathrm{~B} 1950)\left( \pm 0^{\prime \prime} 1\right)$. The continuum and line peaks coincide within 0.05 .

${ }^{\mathrm{b}}$ Single-dish flux scaled from $1.25 \mathrm{~mm}$ (Carico et al. 1992) assuming $\kappa_{v} \propto v^{1.3}$ is $213 \pm 10 \mathrm{mJy}$.

c Equivalent excess blackbody temperature for an emitting region of a 0.3 diameter.

d The total flux density was measured in a 1".2 resolution map, which partially resolves the two nuclei.

Flux density of each nucleus is the sum of CLEAN components, which gives a lower limit.

${ }^{\mathrm{e}}$ Single-dish total flux measured by Radford et al. 1991 is $1038 \mathrm{Jy} \mathrm{km} \mathrm{s}^{-1}$.

${ }^{\mathrm{f}}$ Peak excess brightness temperature of $\mathrm{CO}(2-1)$ line in the channel maps.

${ }^{\mathrm{g}}$ Heliocentric $\mathrm{CO}(2-1)$ velocity $(c z)$ at the nucleus.

${ }^{\mathrm{h}}$ Position angle of the velocity gradient at the nucleus. The last column is for the outer disk.

${ }^{i}$ Line-of-sight velocity width. Our spectrometer does not fully cover Arp $220 \mathrm{~W}$.

${ }^{j}$ The dynamical masses for east and west are those within a radius of 0 ".3 (112 pc) without inclination correction. The total dynamical mass is that within a radius of $250 \mathrm{pc}$ corrected for an inclination of $45^{\circ}$; SYB97.

${ }^{\mathrm{k}}$ See text.

model now appears favored by our new data, as discussed below.

A steep velocity gradient is clearly seen across each nucleus in the U-array velocity map (Fig. $3 b$ ), channel maps (Fig. 1), and also in the IRAM data (DS98). In the CO channel maps, the emission centroid continuously migrates from east of the cross (continuum position of Arp $220 \mathrm{~W}$ ) at lower velocities to west of the cross at high velocities. A similar shift of the emission centroid from southwest to northeast of the nucleus is also seen in Arp $220 \mathrm{E}$; however, it is apparent that the directions of the velocity gradients on $\mathrm{E}$ and $\mathrm{W}$ are not aligned. Moreover, the velocity gradient in Arp $220 \mathrm{~W}$ at P.A. $=263^{\circ}$ is skewed with respect to that in the outer disk (P.A. $=25^{\circ}$, Table 1$)$. The velocity shifts are about $500 \mathrm{~km} \mathrm{~s}^{-1}$ within $0.3(110 \mathrm{pc})$ of the nuclei, as seen in the position-velocity maps (Fig. 4) as well as the channel maps (Fig. 1). (Note that the mean velocities shown in Fig. $3 b$ average over the synthesized beamwidth that includes emission components from both nuclei as well as the larger, P.A. $=25^{\circ}$, disk. The mean velocities therefore grossly underestimate the nuclear velocity gradients. In addition, the actual rotation curve should be determined from the terminal or maximum velocities on each line of sight rather than the mean. The smaller line widths found in SYB97 are due to the loss of low-intensity line wings in the clean component data and the larger 1" beam.)

There have been several centimeter-wave interferometric studies of the velocity field in Arp 220 with somewhat contradictory results. Baan \& Haschick (1995) found a northsouth velocity gradient within the $\mathrm{W}$ nucleus from VLA observations of formaldehyde $\left(\mathrm{H}_{2} \mathrm{CO}\right)$ maser emission, while little velocity gradient was seen in the $\mathrm{E}$ nucleus. In their mean velocity map, the velocity gradient within the $\mathrm{W}$ nucleus is about $\Delta V=125 \mathrm{~km} \mathrm{~s}^{-1}$ in $1^{\prime \prime}$. The true velocity gradient could be larger if measured in a position-velocity map along the line of nodes, although the map was not presented. The velocity gradient at the $\mathrm{W}$ nucleus in the formaldehyde emission is nearly perpendicular to that of CO. VLBI maps of the $\mathrm{OH}$ megamaser emission by Lonsdale et al. (1998) show two components of a few ten parsecs extent in each nucleus. The two in the $\mathrm{W}$ nucleus have $\sim 100 \mathrm{~km} \mathrm{~s}^{-1}$ linewidths, respectively, and are separated by $0.3(110 \mathrm{pc})$ in the north-south direction, but no velocity

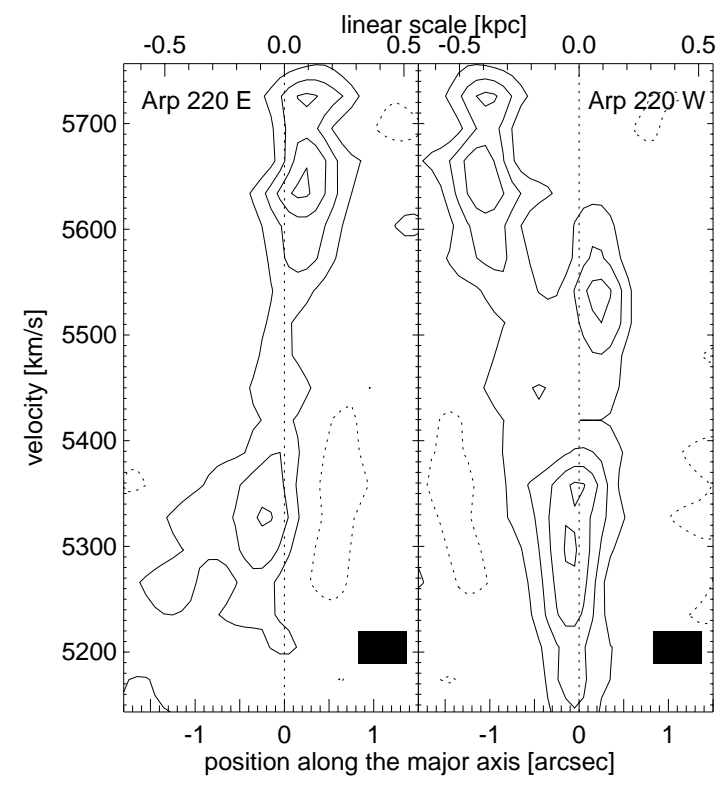

FIG. 4. - CO (2-1) position-velocity maps along the major axes of the nuclear disks. Contours are in steps of $46 \mathrm{mJy}$ beam ${ }^{-1} \mathrm{~km} \mathrm{~s}^{-1}(2 \sigma)$ without zero contours. The filled box in the lower right-hand corner of each panel shows the spatial and velocity resolution. At $-1^{\prime \prime}$ in the P-V map of Arp $220 \mathrm{~W}$, the emission is from Arp $220 \mathrm{E}$, not W. 
gradient is seen between the two components. The two in the $\mathrm{E}$ nucleus are separated by about $50 \mathrm{pc}$ in P.A. $\approx 30^{\circ}$, roughly in accord with the major axis of the east $\mathrm{CO}$ disk $\left(50^{\circ}\right)$. The northeast component is redshifted by about 100 $\mathrm{km} \mathrm{s}^{-1}$ relative to the southwest component, also consistent with the $\mathrm{CO}$ velocity gradient. The two components in each nucleus, however, have strikingly different morphologies; i.e., one is linear with a bright broad-line knot in the middle, and the other is in an amorphous shape. Therefore, the two components in each nucleus do not necessarily indicate the elongation and rotation of a gas disk. Lastly, recent mapping of the H I absorption by Mundell, Ferruit, \& Pedlar (1999) shows a roughly northeast-southwest velocity gradient in the $\mathrm{E}$ nucleus, similar to that found in $\mathrm{CO}$, while velocity structure in the $\mathrm{W}$ nucleus is too complicated to be simply modeled with a velocity gradient. The complicated velocity structure in the $\mathrm{W}$ nucleus could reflect warping of the disk on different scales (since the size of the observed regions ranges by more than 1 order of magnitude) or the selection of favorable gain paths for maser amplification of the nuclear continuum source(s).

\section{NUCLEAR GAS DISKS}

The steep velocity gradients as well as the misalignments of the $\mathrm{CO}$ nuclear disk structures exclude the possibility that the gas is dynamically coherent with the larger disk. Instead, our data suggest that each nucleus has a separate rotating molecular gas disk of diameter about 200 pc (0"6). The directions of the velocity gradients imply that the rotation axes of these two nuclear disks are misaligned. The $\mathrm{W}$ nuclear disk with the major axis in east-west direction was inferred from the crescent morphology of the $2 \mu \mathrm{m}$ emission in HST NICMOS images (Scoville et al. 1998). The sharp falloff of light to the south of the W nucleus in the NICMOS images suggests that the near side (the side of higher extinction) of the west disk is to the south. The NICMOS imagery also resolved the $\mathrm{E}$ nucleus into two peaks separated by 0.4 in the north-south direction, with the southern peak being more highly reddened. It is therefore likely that the near side of the E nuclear disk is also to the south. For these orientations (with the near sides of both disks on the south), the two nuclear disks must be counterrotating. The suggested configuration of the gas disks in Arp 220 is illustrated in Figure 5.

DS98 have interpreted the east and west peaks as starbursting gas clumps formed from gravitational instabilities in the larger gas disk. They interpret the velocity gradients across each nucleus as galactic bar streaming motions or starburst-driven molecular outflows. However, the magnitude of the observed velocity gradients $\left(\sim 500 \mathrm{~km} \mathrm{~s}^{-1}\right)$ and their different position angles across each $\mathrm{CO}$ peak (nearly coincident with the dust continuum, nonthermal radio continuum, and NIR peaks) is strong evidence that these peaks are high-mass concentrations (therefore, probably real galactic nuclei as opposed to starburst luminosity peaks). The velocity range at each nucleus is in fact comparable with the overall linewidth of the $\mathrm{CO}$ over the entire nucleus of Arp 220, and thus the velocity gradients cannot be accounted for as minor perturbations (e.g., streaming) in a bar. Similarly, outflow winds at several hundred $\mathrm{km} \mathrm{s}^{-1}$ would have difficulty remaining molecular, and their expected position angle should be perpendicular to the central disk. (Although the position angle of the velocity gradient at the $\mathrm{W}$ nucleus is almost perpendicular to the

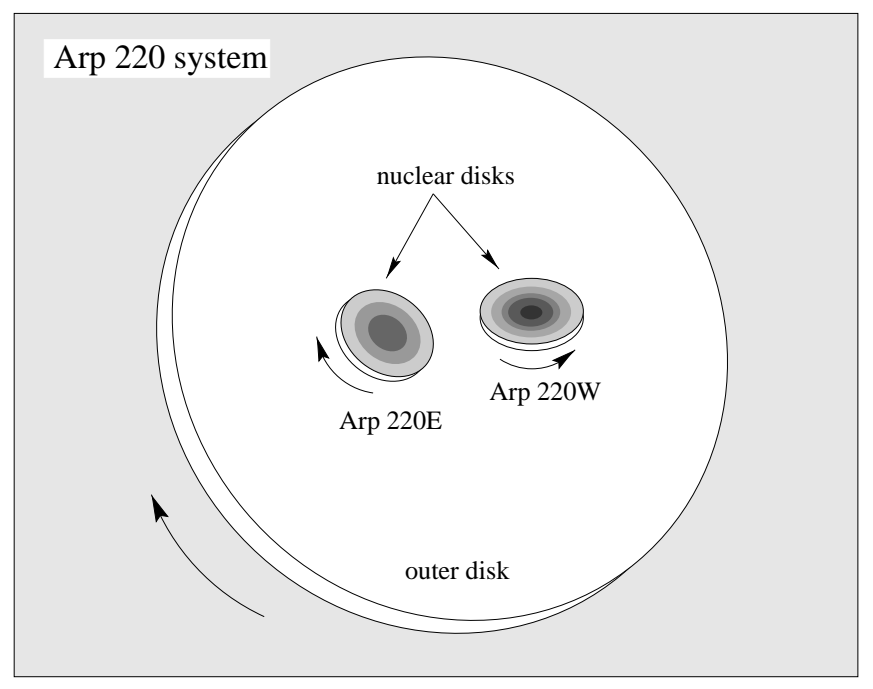

FIG. 5.-Schematic illustration of the Arp 220 disks. The two nuclear disks have radii $\sim 100 \mathrm{pc}$ and misaligned spin axes, probably counterrotating with respect to each other. The dynamical mass within $100 \mathrm{pc}$ radius of each nuclear disk is about $2 \times 10^{9} \sin ^{-2} i M_{\odot}$, where $i$ is the inclination of the disk. Gas masses in the nuclear disks are estimated to be $\sim 10^{9} M_{\odot}$ for each. Each of the nuclei contains a gas disk, young stars formed in the disk, and presumably an old stellar population from the nucleus of the merger progenitor. The stellar component likely has a larger scale height than molecular gas, and thus the gas disk is embedded in the stellar nucleus. Most of the far-IR luminosity of Arp 220 is from the central $100 \mathrm{pc}$ diameter of the nuclear disks. The outer disk has a radius of $\sim 1 \mathrm{kpc}$ formed from the gas from the progenitor galaxies and now rotating around the dynamical center of the merger. It is likely the host of a previous starburst of $\sim 10^{8} \mathrm{yr}$ ago created the $\mathrm{H} \alpha$ bubbles that are $\sim 10 \mathrm{kpc}$ in size.

larger disk and aligned to the $\mathrm{H} \alpha$ double-bubble, the direction of the velocity gradient is opposite to that of the $\mathrm{H} \alpha$ outflow.) Lastly, it is unclear how a starburst-generated outflow could contain a significant fraction of the same dense gas that sustains the starburst.

Whether the mass concentrations seen at the two peaks contain a significant contribution of mass from the premerger galactic nuclei or is almost entirely gas plus young stars is difficult to answer on the basis of existing observational data. The absence of evidence of old stars in the optical/IR spectrum of Arp 220 is certainly not evidence of their absence given the fact that the younger massive stars are much brighter. DS98 argued against a significant mass from the old stellar population on the basis of the mass budgetwhen they add their estimates of the mass in the molecular gas and the young stars required to account for the observed luminosity, this sum approaches the dynamical mass and hence leaves little room for old stars. However, this does not seem to be a strong basis to rule out an older stellar population since the dynamical mass is really a lower limit (due to the uncertain inclination), the molecular mass estimate is clearly uncertain given the extreme excitation conditions, and lastly, the derived young stellar mass depends sensitively on the starburst IMF and the duration of the starbursts (see, e.g., SYB97; Leitherer \& Heckman 1995).

\subsection{Comparison of Dust and CO Emission}

The marked difference in the extent of $\mathrm{CO}$ and continuum emission is real, not an artifact of missing flux in the continuum map. The LHU-array CO map made with all 
channels combined before Fourier transform (i.e., processed in the same way as continuum maps) still shows the $\mathrm{CO}$ emission extended well outside the double nuclei. The striking difference between the $\mathrm{CO}$ and dust spatial extents could be due to the following: (1) line-of-sight blocking of the $\mathrm{CO}$ emission (to suppress that from the nuclear regions); (2) different dependences of the $\mathrm{CO}$ and dust emissivities on the physical conditions (principally temperature and column density); or, (3) a reduction in the $\mathrm{CO}$ abundance relative to the dust in the ISM of the nuclear clusters. The idea of overlapping CO emission clouds was proposed for the larger gas disk of Arp 220 by Downes, Solomon, \& Radford (1993), and this idea could be extended to postulate that there is even more severe overlap in the compact nuclear disks than in the larger disk. If the gas cloud density in each nuclear disk is so high that the clouds overlap both on the sky and in line-of-sight velocity, then the intensity of the optically thick $\mathrm{CO}$ will reflect the gas temperature at the disk surface in the nearest cloud at each velocity. (To be a significant effect, it is required that the stars in the disk dominate the potential of the gas; otherwise, if the gas actually contributes most of the mass, increases in the mass surface density of the disk cause comparable increases in the $\mathrm{CO}$ line width, and hence yield increased velocityintegrated emission.)

Alternatively, if the dust and $\mathrm{CO}$ emission had different dependences on the physical conditions, which are likely to be functions of radius, then the dust continuum could be more centrally peaked than the CO. The optically thin dust emission is proportional to the product of the dust temperature and column density, while the $\mathrm{CO}$ surface brightness (assuming the $\mathrm{CO}$ is optically thick) will vary as a product of (1) the $\mathrm{CO}$ column density; (2) a factor proportional to the ratio $T_{\mathrm{ex}} /\left\langle n_{\mathrm{H}_{2}}\right\rangle^{1 / 2}$, where $T_{\mathrm{ex}}$ is the excitation temperature and $\left\langle n_{\mathrm{H}_{2}}\right\rangle$ is the mean $\mathrm{H}_{2}$ density; and (3) a geometry-dependent factor that reflects the importance of external gravitational potentials and pressures in the emission-line width and cloud binding (see, e.g., Downes et al. 1993; Bryant \& Scoville 1996). Thus even with a constant ratio of the $\mathrm{CO}$ and dust abundances, a dense ISM like that in the nuclear disks of Arp 220 might naturally have relatively strong, optically thin dust emission if temperature and density increases at small radii without increasing $T_{\text {ex }} /\left\langle n_{\mathrm{H}_{2}}\right\rangle^{1 / 2}$.

Finally, a low CO-to-continuum ratio near the nuclei could occur if the $\mathrm{CO}$ is optically thin due to high temperatures (DS98), or the gas is depleted relative to the dust in the vicinity of the nuclei. However, since the observed $\mathrm{CO}$ brightness temperatures $\left(T_{b} \sim 38 \mathrm{~K}\right)$ are comparable to the mean dust temperature $(42 \mathrm{~K})$ derived from fitting the FIR SED, low opacity in the CO lines would necessitate that the gas is much hotter and not in thermal equilibrium with the dust (despite the general expectation that they should be in thermal equilibrium at densities $\geq 10^{4}-10^{5}$ $\mathrm{cm}^{-3}$ ). In addition, the CO 2-1/1-0 line ratios indicate that the lower $\mathrm{CO}$ transitions cannot be optically thin. It is therefore unlikely that the bulk of the $\mathrm{CO}$ emission from the nuclear gas disks is optically thin.

It is useful to review the necessity of continuum subtraction for the situation where $\mathrm{CO}$ emission is optically thick and the clouds are overlapping. Subtraction of the continuum from the emission line channels is appropriate if, at each velocity, the optically thick $\mathrm{CO}$ covers only a small fraction of the projected area of the continuum source. On the other hand, if the continuum source is embedded in and totally covered by the optically thick $\mathrm{CO}$ emission at each velocity, then continuum subtraction should be done only outside the velocity range of the $\mathrm{CO}$ line. The $\mathrm{CO}$ fluxes of the nuclear disks (Table 1) were measured from continuumsubtracted data, and thus underestimate the true $\mathrm{CO}$ flux and brightness temperature in the latter case. Without continuum subtraction, the total $\mathrm{CO}$ flux and the peak brightness temperature of the nuclear gas disks are $43 \mathrm{~K}$ and 150 $\mathrm{Jy} \mathrm{km} \mathrm{s}{ }^{-1}$ for Arp $220 \mathrm{E}$, and $48 \mathrm{~K}$ and $260 \mathrm{Jy} \mathrm{km} \mathrm{s}^{-1}$ for Arp $220 \mathrm{~W}$-these flux values are about 30\% larger than those given in Table 1 . The real situation is probably between these extreme cases; i.e., the continuum emission is partially covered by optically thick gas, but is closer to the low-covering situation since only a small fraction of the full line width is represented along each line of sight to the continuum source. In any case, the continuum subtraction does not affect the velocity gradients seen in the nuclear disks since similar gradients are seen in both the continuum subtracted and nonsubtracted line maps.

\subsection{Gas and Dynamical Masses of the Nuclear Disks}

The dynamical mass in each nuclear disk within 100 pc radius is $\sim 2 \times 10^{9} \sin ^{-2} i M_{\odot}$, where $i$ is the inclination of the disk (see Table 1). A thin-disk model with parameters of $V_{\text {rot }} \sin i \sim 265 \pm 10 \mathrm{~km} \mathrm{~s}^{-1}$ at $r_{\text {peak }} \sim 0$.'25 produced a reasonably good fit to the position-velocity diagram of Arp 220 E. The presence of the two nuclear disks, their misaligned rotational axes, and their large masses strongly implies that the two peaks in millimeter, radio, and NIR are major mass concentrations (i.e., galactic nuclei), and that Arp 220 is indeed a merger. The two nuclear disks are embedded in an outer gas disk of kiloparsec size. The dynamical mass within the orbit of the two nuclei (i.e., $r<250 \mathrm{pc}$ ) was estimated to be $5.4 \times 10^{9} M_{\odot}$ by SYB97. Thus more than half of the mass in this region belongs to the two nuclei, although the dynamical masses have large uncertainties due to the uncertain inclinations.

Estimation of the molecular gas mass from the observed $\mathrm{CO}$ emission in the nuclear disks of Arp 220 is extremely uncertain. The physical conditions of the gas are undoubtedly very different from those of the Galactic giant molecular clouds (GMCs), where the "standard" Galactic CO-to- $\mathrm{H}_{2}$ conversion factor is derived. Moreover, it has been pointed out by Downes et al. (1993) that if the largescale gravitational field of the galactic nucleus (rather than the self-gravity of individual clouds) dominates the structure of the nuclear $\mathrm{CO}$ emission regions, the escape probability of optically thick CO line photons will be increased (because of the larger velocity width), thus reducing the CO-to- $\mathrm{H}_{2}$ conversion factor. SYB97 estimated an average CO-to- $\mathrm{H}_{2}$ conversion factor of 0.45 times the Galactic value for the larger molecular disk in Arp 220. Their method was based on fitting the observed line profiles to an axisymmetric disk model and assuming that the enclosed molecular gas mass determined the disk rotation velocity as a function of radius. This average conversion factor is probably inapplicable to the very compact nuclear gas disks because of the more extreme physical conditions and the likely contribution of stars to the mass within the nuclei.

Here we follow the discussion in the Appendix of Bryant \& Scoville (1996) to place constraints on the molecular gas mass. A firm lower limit of $1 \times 10^{8} M_{\odot}$ for Arp $220 \mathrm{~W}$ (and two-thirds as much for Arp $220 \mathrm{E}$ ) is obtained if the $\mathrm{CO}$ is 
optically thin and in LTE at $40 \mathrm{~K}$ with a CO-to- $\mathrm{H}_{2}$ abundance of $10^{-4}$. For optically thick $\mathrm{CO}$ emission from selfgravitating GMCs, the higher gas temperature in Arp 220 can be compensated to some extent by the higher gas density, since the conversion factor scales as the ratio $\left\langle n_{\mathrm{H}_{2}}\right\rangle^{1 / 2} / T_{b}$. If the $T_{b}$ is 50 and $10 \mathrm{~K}$ in the Arp 220 and Galactic GMCs, respectively, and the mean volume densities are $10^{4.5}$ and $300 \mathrm{~cm}^{-3}$, then the conversion factor is changed (actually increased) by only a factor of 2 because of the compensating changes in temperature and density. The higher gas density is implied by the observations of $\mathrm{HCN}$, a tracer of dense gas. The HCN-to-CO line ratio in Arp 220 requires a mean density exceeding $10^{4} \mathrm{~cm}^{-3}$ (Solomon, Downes, \& Radford 1992). In addition, this density is consistent with the assumption that the mass derived below is distributed in a disk of $100 \mathrm{pc}$ radius and a thickness of $\sim 30$ pc. By modeling the effects of the galactic nucleus gravitational potential as a disk, there are two possibilities: an entirely self-gravitating gas disk, and a gas disk embedded in a potential predominantly due to stars. If the gas disk is geometrically thin (with inclination $i$ ), smooth, and selfgravitating, then the gas mass in Arp $220 \mathrm{~W}$ is $1.3 \times 10^{9}$ sec $i M_{\odot}$ for gas with density and brightness temperature having a ratio of $\left\langle n_{\mathrm{H}_{2}}\right\rangle^{1 / 2} / T_{b}$, similar to Galactic GMCs. For an inclination of $45^{\circ}$, the conversion factor in the disk is then 0.4 times the Galactic value, and the gas masses in east and west are (1-2) $\times 10^{9} M_{\odot}$, which agrees within a factor of 2 to the values derived by DS98 using the similar method of Downes et al. (1993). If the gas disk is not entirely self-gravitating but bound partly by the stellar (or central black hole) gravity, then the gas mass in $\mathrm{W}$ is 1.6 $\times 10^{9} f_{\mathbf{p}}^{1 / 2} \sec i M_{\odot}$, where $f_{p}(\ll 1)$ is a parameter describing the relative contribution of gas and stars to the kinematics of the disk. (Specifically, $f_{p}$ is the ratio of gaseous to stellar mass densities at the midplane of the disk; see Bryant \& Scoville 1996 for details. Our observations do not constrain this parameter, and thus the gas mass cannot be evaluated in this case.) A firm upper limit of gas mass is $M_{\mathrm{gas}} \leq M_{\mathrm{dyn}}$.

\section{MERGER EVOLUTION AND LUMINOSITY SOURCE}

Numerical simulations of mergers (see, e.g., Barnes \& Hernquist 1991) have shown that the gas concentrates at the center of each galaxy in the early phases of interaction, with some delay after the first encounter when progenitors have massive bulges (Mihos \& Hernquist 1994). Later, the gas condensations merge and form a single gas disk as they approach each other. Our results are largely consistent with this scenario since the larger gas disk extending to a $1 \mathrm{kpc}$ radius has $70 \%$ of $\mathrm{CO}$ luminosity. However, it is clear from the results presented here that each nucleus also has its own smaller, denser disk, which survives until the merging is nearly complete. These compact nuclear disks are probably remnants of the gas that concentrated within the nuclei when they were much further apart. Presumably any gas concentrated within the deep potentials of the original nuclei where the escape velocity is $\geq 300 \mathrm{~km} \mathrm{~s}^{-1}$ would be trapped until it is either exhausted in star formation or AGN accretion, or is ejected in a more diffuse wind generated by the starburst. The latter would probably be inhibited as a major mechanism of mass loss until the disk has evolved to a much lower column density than is presently the case. As long as there is enough cold dense gas in the disk to dissipate and radiate the wind kinetic energy, the outflows generated by starburst activity embedded within the disks cannot rip much molecular gas off the disks.

Our observations do not give a conclusive answer regarding the origin of the large FIR luminosity of Arp 220, since the dust temperature is consistent with the dominant heating source being either a starburst or an AGN. However, our results do give the following spatial restriction on the energy source. The fact that the $1 \mathrm{~mm}$ continuum fits on the FIR tail of the overall spectral energy distribution (containing most of the bolometric luminosity) suggests that spatial distribution of the energy source(s) is similar to that of the $1 \mathrm{~mm}$ continuum, i.e., dominated by two components, with the western source being probably twice as luminous. The size of the two luminosity sources must also be $\lesssim 100$ pc. It is possible for additional components to contribute to the bolometric luminosity (without contributing $1 \mathrm{~mm}$ flux) only if the temperatures of these components are much higher than for the sources at the nuclei. Since it seems unlikely that dust in the outer gas disk should have higher temperatures than that in the nuclei, we conclude that only a small portion of the luminosity originates from the $1 \mathrm{kpc}$ disk. (We therefore differ from DS98, who attribute half of the luminosity to the outer molecular disk, but as noted earlier, claim a larger fraction of the $1 \mathrm{~mm}$ continuum in an extended component.)

The suggested distribution of luminosity is consistent with either a double starburst or double AGN for the energy source. It excludes a single dominant AGN or a starburst in the gas concentration toward the dynamical center between the two nuclei. Since the energy sources are in both nuclei, the elevated luminosity of Arp 220 was probably triggered by gas condensation within the nuclei of the progenitor galaxies. The upper limit of $100 \mathrm{pc}$ is comparable to the spatial extent of the unresolved VLBI sources, probably radio supernovae, around the $\mathrm{W}$ nucleus (Smith et al. 1998). If the bulk of the luminosity of Arp 220 arises from starbursts (see, e.g., Sturm et al. 1996; Lutz et al. 1996; Genzel et al. 1998, and references therein), the starbursts are embedded in the two nuclear gas disks and their radiation would be highly extincted. The average extinction perpendicular to the disks is $A_{V} \sim 1000$ mag based on the mean gas and dust surface densities. Armus et al. (1995) suggested based on NIR photometry and spectroscopy (CO absorption and $\mathrm{Br} \gamma$ ) that there are young (several $10^{6} \mathrm{yr}$ ) starbursts at the two nuclei surrounded by an old $\left(\sim 10^{8} \mathrm{yr}\right)$ starburst region of $\sim 5^{\prime \prime}(2 \mathrm{kpc})$ diameter. In their accounting, these starbursts contribute less than $10 \%$ to the bolometric luminosity of Arp 220; however, they adopted $10 \mathrm{mag}$ of visual extinction in a foreground screen, whereas the ISO observations indicate probably at least $45 \mathrm{mag}$ for a screen model, or $A_{V} \sim 1000 \mathrm{mag}$ for a mixed source/dust model (Lutz et al. 1996). The older starburst appears to have occurred in the outer kiloparsec-size molecular disk and probably caused the large $\mathrm{H} \alpha$ double-bubble whose dynamical age is $\sim 10^{8}$ yr (Heckman et al. 1990). The current starbursts in the nuclear disks suffer from large extinction, and thus could contribute more to the bolometric luminosity than the above estimate from NIR observations.

Another restriction for the luminosity source is the average luminosity-to-mass ratio $L_{\mathrm{IR}} / M_{\mathrm{dyn}}$ in the nuclear disks. It is as high as $200 L_{\odot} / M_{\odot}$ for nominal inclinations of $45^{\circ}$. Such a high ratio cannot be attained unless the initial mass function of the starbursts is biased toward high masses 
(e.g., truncated below a few $M_{\odot}$ ), or there are additional energy sources such as heavily obscured AGNs (SYB97; Shier, Rieke, \& Rieke 1996). The large mass of gas in the nuclear disks can fuel both starbursts and AGNs for $\geq 10^{8}$ $\mathrm{yr}$, whereas the rotational kinetic energy of the nuclear disks $\left(\approx 10^{50} J\right.$ for each) can sustain the FIR luminosity for just $10^{4} \mathrm{yr}$. Assuming that the nucleus is not viewed close to the polar axis, the high opacities of the nuclear ISM can easily absorb the direct emission of AGNs (if any exist) from moderately high-energy X-ray to mid-IR wavelengths. The lack of a compact, high-brightness temperature nuclear radio source also does not rule out an AGN, since not all AGNs have strong radio emission (Smith et al. 1998). Lonsdale et al. (1998) pointed out that the linear morphology and broad linewidth of one of the two $\mathrm{OH}$ megamaser components in each nucleus could be due to twin jets and a molecular torus of an AGN, although they did not exclude the possibility of starburst-driven shocks for the excitation mechanism of the $\mathrm{OH}$ maser.

\section{CONCLUSIONS}

Our 0.5 resolution observations of $\mathrm{CO}$ and dust continuum provide the following picture of the central region of
Arp 220: there are two counterrotating gas (and dust) disks of $\sim 100 \mathrm{pc}$ radius within each of the double nuclei. This counterrotation may reflect the geometry of the progenitor galactic disks in their mutual encounter. If so, it would facilitate the eventual merging of the galaxies because of the more effective cancellation of angular momentum and dissipation of kinetic energy in such encounters. These nuclear disks are embedded in a kiloparsec-size gas disk, which rotates around the dynamical center of the merger. The gas and dust in the nuclear disks is hot $(\geq 40 \mathrm{~K})$, dense, and probably covers at least half of the nuclear-disk surface area. The dynamical masses within a $100 \mathrm{pc}$ radius of each nucleus are $\gtrsim 2 \times 10^{9} M_{\odot}$. The FIR luminosity is generated predominantly within the central $100 \mathrm{pc}$ of the two nuclei with a high luminosity-to-mass ratio.

We are grateful to the OVRO staff for their hard work that led to the smooth startup of the new configuration. We thank Carole Mundell for kindly communicating the results of $\mathrm{H}$ I observations prior to publication. The OVRO millimeter array is funded by NSF grant AST 96-13717 and the K. T. and E. L. Norris Foundation. K. S. is supported by a JSPS fellowship.

\section{REFERENCES}

Armus, L., Neugebauer, G., Soifer, B. T., \& Matthews, K. 1995, AJ, 110, 2610

Arp, H. 1966, Atlas of Peculiar Galaxies (Pasadena: California Inst. Tech.)

Baan, W. A., \& Haschick, A. D. 1995, ApJ, 454, 745

Barnes, J. E., \& Hernquist, L. E. 1991, ApJ, 370, L65

Bryant, P. M., \& Scoville, N. Z. 1996, ApJ, 457, 678

Carico, D. P., Keen, J., Soifer, B. T., \& Neugebauer, G. 1992, PASP, 104, 1086

Downes, D., \& Solomon, P. 1998, ApJ, 507, 615

Downes, D., Solomon, P., \& Radford, S. J. E. 1993, ApJ, 414, L13

Genzel, R., et al. 1998, ApJ, 498, 579

Graham, J. R., Carico, D. P., Matthews, K., Neugebauer, G., Soifer, B. T., \& Wilson, T. D. 1990, ApJ, 354, L5

Heckman, T. M., Armus, L., \& Miley, G. K. 1987, AJ, 92, 276

.1990, ApJS, 74, 833

Larkin, J. E., Armus, L., Knop, R. A., Matthews, K., \& Soifer, B. T. 1995, ApJ, 452, 599

Leitherer, C., \& Heckman, T. 1995, ApJS, 96, 9

Lonsdale, C. J., Diamond, P. J., Smith, H. E., \& Londale, C. J. 1998, ApJ, 493, L13

Lutz, D., et al. 1996, A\&A, 315, L137

Mihos, J. C., \& Hernquist, L. 1994, ApJ, 431, L9

Mundell, C. G., Ferruit, P., \& Pedlar, A. 1999, in preparation
Norris, R. P. 1985, MNRAS, 216, 701

Radford, S. J. E., Solomon, P. M., \& Downes, D. 1991, ApJ, 368, L15

Sanders, D. B., Scoville, N. Z., \& Soifer, B. T. 1991, ApJ, 370, 158

Sanders, D. B., Soifer, B. T., Elias, J. H., Madore, B. F., Matthews, K., Neugebauer, G., \& Scoville, N. Z. 1988, ApJ, 325, 74

Scoville, N. Z., Carlstrom, J. E., Chandler, C. J., Phillips, J. A., Scott, S. L., Tilanus, R. P. J., \& Wang, Z. 1993, PASP, 105, 1482

Scoville, N. Z., et al. 1998, ApJ, 492, L107

Scoville, N. Z., Sanders, D. B., Sargent, A. I., Soifer, B. T., Scott, S. L., \& Lo, K. Y. 1986, ApJ, 311, L47

Scoville, N. Z., Sargent, A. I., Sanders, D. B., \& Soifer, B. T. 1991, ApJ, 366, L5

Scoville, N. Z., Yun, M. S., \& Bryant, P. M. 1997, ApJ, 484, 702 (SYB97)

Shier, L. M., Rieke, M. J., \& Rieke, G. H. 1996, ApJ, 470, 222

Smith, H. E., Lonsdale, C. J., Lonsdale, C. J., \& Diamond, P. J. 1998, ApJ, 493, L17

Soifer, B. T., Sanders, D. B., Madore, B. F., Neugebauer, G., Danielson, G. E., Elias, J. H., Lonsdale, C. J., \& Rice, W. L. 1987, ApJ, 320, 238

Solomon, P. M., Downes, D., \& Radford, S. J. E. 1992, ApJ, 387, L55

Solomon, P. M., Downes, D., Radford, S. J. E., \& Barrett, J. W. 1997, ApJ, 478,144

Sturm, E., et al. 1996, A\&A, 315, L133

Taniguchi, Y., \& Shioya, Y. 1998, ApJ, 501, L167 